\title{
Optimiser l'usage des cartes conceptuelles dans l'apprentissage par problèmes (APP) dans le cursus préclinique : le point de vue des enseignants
}

\author{
A Teacher's Perspective on How to Optimize the Use of Concept Maps \\ in Problem-Based Learning (PBL) at the undergraduate level.
}

\author{
Nathalie CAIRE FON ${ }^{1}$, Béatrice PUDELKO ${ }^{2}$, Marie-Claude AUDÉTAT ${ }^{3}$ \\ 1 Département de médecine de famille et médecine d'urgence, Faculté de Médecine, Université de Montréal, Montréal, \\ Canada \\ 2 Département Éducation, et Centre de recherche «Laboratoire en informatique cognitive et environnements de formation » \\ (LICEF) - Téluq-Université du Québec, Montréal, Canada \\ 3 Département de médecine de famille et médecine d'urgence et Centre de pédagogie appliquée aux sciences de la santé \\ (CPASS)- Faculté de Médecine, Université de Montréal, Montréal, Canada, et Unité des internistes généralistes et \\ pédiatres (UIGP) et UDREM, Faculté de Médecine, Université de Genève, Suisse
}

Manuscrit reçu le 16 mai 2016 ; commentaires éditoriaux formulés aux auteurs le $1^{\mathrm{er}}$ octobre 2016 ; accepté pour publication le $1^{\mathrm{er}}$ novembre 2016

\begin{tabular}{|c|c|}
\hline $\begin{array}{l}\text { Mots-clés } \\
\text { carte conceptuelle ; } \\
\text { apprentissage } \\
\text { par problèmes ; } \\
\text { guidance ; } \\
\text { connaissances } \\
\text { biomédicales ; } \\
\text { organisation des } \\
\text { connaissances ; } \\
\text { connaissances } \\
\text { causales }\end{array}$ & $\begin{array}{l}\text { Résumé - Problématique : La carte conceptuelle (CC) est une stratégie d'apprentissage popu- } \\
\text { laire en éducation des sciences de la santé. Elle est notamment utilisée pour soutenir l'orga- } \\
\text { nisation des connaissances, dans l'apprentissage par problème (APP) en formation médicale } \\
\text { pré-clinique. On dispose cependant de peu de données sur la meilleure façon de l'utiliser dans } \\
\text { ce contexte pédagogique. But : Cette étude exploratoire visait à déterminer si les responsables } \\
\text { des cours d'APP de la formation médicale au pré-clinique partagent une conception commune } \\
\text { des objectifs pédagogiques de la construction des CC et de la nature et de l'organisation des } \\
\text { connaissances qui devraient être représentées dans la CC pour favoriser l'atteinte des objectifs } \\
\text { visés. Méthodes : Cette recherche est une étude de cas interprétative, à laquelle ont participé } \\
\text { huit enseignants responsables des cours d'APP. Chaque participant devait construire une CC } \\
\text { à partir d'un cas-problème typique de son cours. Le recueil de données s'est fait au travers de } \\
\text { l'enregistrement vidéo de la verbalisation concomitante durant la séance de construction de la } \\
\text { CC, l'observation directe et le recueil des traces de l'activité et l'entretien semi-dirigé de } \\
\text { groupe. L'analyse des données a consisté en une analyse thématique de contenu des données } \\
\text { verbales et des traces écrites et graphiques recueillies. Résultats : Selon les participants, }\end{array}$ \\
\hline
\end{tabular}


Keywords concept map; problem-based learning; scaffolding; biomedical knowledge; knowledge organization; causal knowledge l'objectif pédagogique des CC est de favoriser la construction d'une représentation cohérente des mécanismes causaux permettant d'expliquer le problème clinique du cas. Les connaissances biomédicales sont représentées dans la CC sous forme d'une structure causale reliant la pathologie aux données cliniques du cas, regroupées en « catégories cliniques ». Des conceptions pédagogiques partagées sur la façon d'optimiser l'usage des CC dans l'APP ont été identifiées. Conclusion Notre recherche a permis de formuler des recommandations pour favoriser un meilleur alignement pédagogique de la construction des $\mathrm{CC}$ et des objectifs pédagogiques visés dans le cadre de l'APP.

Abstract - Background: Concept mapping is frequently used in health sciences as a teaching tool for organizing knowledge. It has been used in a variety of medical-education settings including problem-based learning. However, there are few recommendations regarding how to optimize its use in this particular learning context. Purpose: This study examined how undergraduate medical-education teachers perceived concept mapping in problem-based learning (PBL) courses. Our goal was to determine if these teachers shared a common understanding of: (1) concept-mapping learning outcomes (2) the nature and structure of knowledge to be represented in the conceptual maps given these learning outcomes. Methods: Eight teachers responsible for PBL undergraduate courses participated in this interpretive case study. Based on a case problem used in their own course, participants were asked to construct a concept map. During the process they were required to think aloud. The activity was followed by a semi-directed group interview. Both activities were videotaped and fully transcribed. Verbal and written data were analyzed using a thematic content analysis. Results: Participants felt concept mapping should allow students to construct a coherent representation of the causal mechanisms explaining the case problem. Biomedical knowledge was represented by participants as causal networks linked to the case-problem clinical data. Clinical data were organized in broader clinical categories such as signs, symptoms, side effects, etc. Participants expressed similar conceptions of their intended learning outcomes and concept mapping uses. Conclusion: Our results allow us to make some recommendations about achieving a constructive alignment in PBL courses between concept-mapping activity and intended learning outcomes.

\section{Problématique}

La carte conceptuelle (CC) fait partie des outils de représentation externe des connaissances tels que l'écriture, les diagrammes ou les cartes géographiques, combinant l'encodage symbolique et l'organisation spatiale des informations. Outre leur fonction de préservation des connaissances sur un support externe, ces outils ont aussi pour fonction de faciliter les processus de traitement des connaissances aussi bien à la phase de la construction qu'à la phase de l'utilisation des représentations construites ${ }^{[1]}$.

La CC est une représentation externe des connaissances sous forme de réseau composé de « nœuds » représentant les concepts, et de liens représentant les relations entre ces concepts. En éducation, elle est considérée comme un « outil cognitif » favorisant l'apprentissage signifiant, c'est-à-dire une compréhension en profondeur des connaissances conceptuelles complexes, mais aussi comme un « outil métacognitif », visant à développer la capacité d'autorégulation de ses apprentissages ${ }^{[1]}$.

La théorie et la méthode des CC élaborées par Novak $^{[2]}$ sous-tendent la grande majorité des usages des représentations du type "réseau » dans les domaines scientifiques, du primaire au postsecondaire $^{[3]}$. C'est aussi le cas des sciences de la santé, où la $\mathrm{CC}$ est utilisée principalement au niveau du cursus préclinique, surtout en médecine et en sciences infirmières ${ }^{[4,5]}$. 
La plupart des études réalisées dans les domaines de la santé évaluent positivement l'apport des CC à l'apprentissage ${ }^{[6]}$. Toutefois, les résultats de l'analyse critique de Pudelko et al. ${ }^{[4]}$ soulignent que, malgré l'abondance des recherches, il existe encore peu de données démontrant de façon probante les effets positifs de la construction des CC sur l'apprentissage. Pour expliquer ce constat, ces chercheurs avancent l'hypothèse d'un manque d'alignement de la structure de la $\mathrm{CC}$ aux objectifs pédagogiques dans une situation d'apprentissage donnée. En effet, la CC est utilisée pour une grande variété d'objectifs pédagogiques, allant de la compréhension des textes jusqu'au développement de la pensée critique ou de l'autorégulation des processus d'apprentissage. Mais la façon dont les caractéristiques de l'activité représentationnelle avec la CC permettent de soutenir l'atteinte de chacun de ces objectifs est rarement analysée ou mise en rapport avec les particularités des domaines étudiés et les caractéristiques des étudiants. Autrement dit, peu d'attention est accordée aux conditions nécessaires pour réaliser un alignement pédagogique satisfaisant (constructive alignment ${ }^{[7]}$ ) garantissant une cohérence entre les activités d'apprentissage avec la CC, les objectifs visés et les stratégies d'évaluation dans une situation d'apprentissage donnée.

Dans le cadre de l'apprentissage par problèmes (APP), adopté par de nombreux programmes de formation préclinique dans les facultés de médecine, diverses formes de soutien à l'apprentissage sont proposées aux étudiants, parmi lesquelles les représentations externes des connaissances telles que les $\mathrm{CC}^{[8]}$. Cependant, à l'instar d'autres types de représentations externes, les $\mathrm{CC}$ restent encore peu étudiées et les résultats des études existantes ne permettent pas de faire des recommandations permettant de les aligner aux objectifs pédagogiques qui peuvent être visés dans le cadre de l'APP ${ }^{[9]}$.

\section{Contexte}

Depuis 1993, la Faculté de médecine de l’Université de Montréal utilise l'APP inspirée de la méthode en sept étapes proposée par l'Université de
Maastricht ${ }^{[10]}$ pour la formation des étudiants de niveau préclinique. Ces étapes sont regroupées en trois phases : la première ( «l'aller ») et la dernière («le retour »), consistent en des séances de groupe animées par un tuteur. Entre les deux se trouve la phase de l'étude des recueils de référence, réalisée individuellement par chaque étudiant.

En 2004, la construction de CC selon l'approche de Novak a été intégrée à l'APP à titre de stratégie d'apprentissage active, favorisant l'organisation des connaissances par les étudiants. L'utilisation du logiciel CMap Tools ${ }^{[11]}$ conçu pour soutenir la construction des CC selon la méthode de Novak, a alors été recommandée. Des activités de formation à la construction des $\mathrm{CC}$ avec $\mathrm{Cmap}$ Tools et à leur usage dans le cadre de l'APP sont offertes à tous les enseignants impliqués dans l'APP ainsi qu'aux étudiants.

La démarche de l'APP soutenue par l'activité des CC est réalisée de la façon suivante :

- Lors de «l'aller», les étudiants analysent en groupe un nouveau cas clinique. Le tuteur les encourage à élaborer des hypothèses pour expliquer les phénomènes illustrés par le cas. Le groupe doit représenter, dans une «CMap» (une CC construite avec CMap Tools), les phénomènes à expliquer, puis les relier de façon à construire une représentation de l'ensemble du problème. Cette CMap dite «naïve » peut contenir des liens ou des notions inexactes ou incomplètes.

- L'étude individuelle doit permettre aux étudiants de développer leurs connaissances en lien avec les objectifs d'apprentissage. Grâce aux notions nouvellement acquises, les étudiants sont encouragés à corriger, à compléter ou à refaire la carte «naïve » de façon individuelle.

- Lors du «retour» les étudiants reviennent en groupe pour mettre en commun leurs connaissances, sous la supervision du tuteur. Le problème est réexpliqué et la carte «naïve » est corrigée et enrichie par le groupe. $\mathrm{La} \mathrm{CC}$ finale doit permettre une synthèse du problème et des notions scientifiques fondamentales qui s'y rattachent. Elle doit être exempte d'erreurs, par exemple des liens propositionnels erronés.

Les commentaires des tuteurs sur les CC s'avèrent plutôt positifs, notamment parce qu'elles leur 


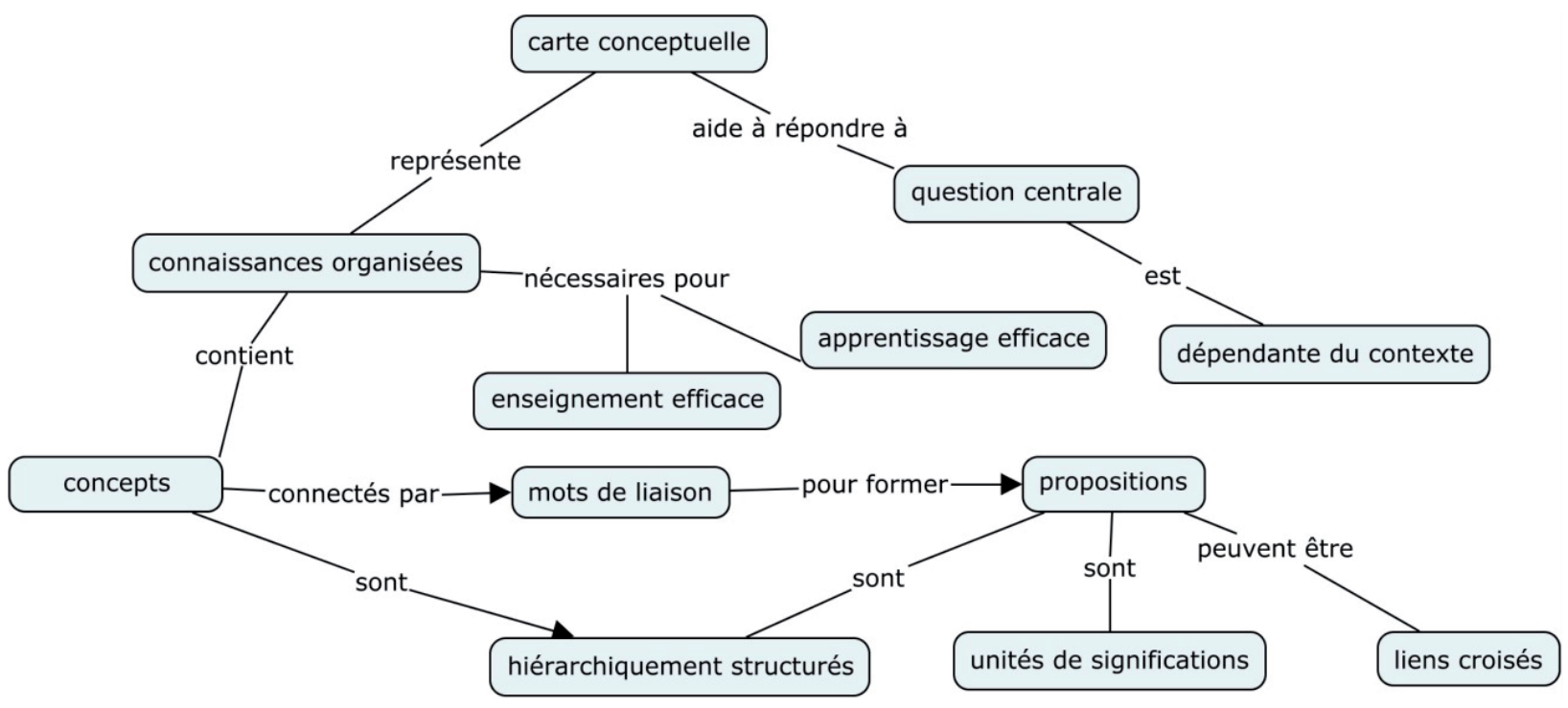

Fig. 1. La carte conceptuelle selon Novak (répéré et adapté du site Cmap Tools, http://cmap.inmc.us/docs/concept-map).

permettent de formuler des rétroactions spécifiques et d'identifier des étudiants en difficulté. Nos observations indiquent que les tuteurs sont capables de reconnaître intuitivement une «bonne carte », sans être pour autant capable d'expliquer les raisons de leur jugement. De plus, ils semblent divisés sur la nature des connaissances à représenter dans la CC. Ils s'entendent pour dire que la CC devrait être construite à partir des données du cas-problème, mais pour certains, elle devrait représenter l'ensemble des connaissances biomédicales en lien avec la maladie présentée alors que pour d'autres elle devrait représenter uniquement les données cliniques du cas-problème. Des questions récurrentes des tuteurs et des étudiants portent ainsi sur la nature de la «bonne carte » et donc de la «bonne » organisation des connaissances et sur son degré de détail. Cette dernière question a d'ailleurs déjà été identifiée comme un obstacle à l'utilisation des CC comme stratégie pédagogique $^{[12]}$. Il est possible que les tuteurs demeurent incertains quant à la nature de l'organisation des connaissances dans la carte parce qu'ils ne disposent pas d'une représentation partagée des objectifs pédagogiques précis de la stratégie de la CC.

\section{Cadre conceptuel}

Dans l'approche élaborée par Novak ${ }^{[2]}$, construire une $\mathrm{CC}$ consiste à représenter les connaissances sous forme d'un réseau propositionnel hiérarchique. La CC est une représentation externe dite « indépendante du domaine » car son utilisation vise à faciliter les processus cognitifs impliqués dans la compréhension des connaissances scientifiques dans différents domaines. Novak postule que l'apprentissage est favorisé lorsque l'apprenant relie les concepts les plus généraux d'un domaine aux concepts plus spécifiques. L'ensemble de propositions, constituées chacune de deux concepts reliés par un lien, forme une structure hiérarchique orientée de façon « descendante », dans laquelle les concepts plus généraux situés en haut de la carte sont reliés aux concepts plus spécifiques situés en bas de la carte. La méthode de Novak ${ }^{[10]}$ offre un ensemble de consignes sur la façon de construire un réseau hiérarchique propositionnel en partant d'un ensemble de concepts. Le logiciel CMap Tools présente des fonctionnalités qui facilitent la construction des CC selon cette méthode (voir figure 1). 
L'utilisation des CC pour des objectifs aussi divers que le soutien à l'apprentissage signifiant, à la mémorisation, à l'autorégulation des apprentissages et au développement de la pensée critique, indique le caractère polyvalent de la représentation des connaissances avec la CC. Mais en même temps, cette polyvalence soulève la question de la façon dont l'activité d'apprentissage avec la CC devrait être adaptée pour favoriser l'atteinte de ces différents objectifs. D'une part, on peut se demander si la seule structuration des connaissances sous la forme d'une CC hiérarchique permet de représenter adéquatement les connaissances dans le domaine concerné pour un objectif pédagogique donné. D'autre part, il s'agit de décider si les consignes de la méthode de Novak offrent un soutien suffisant pour favoriser les processus cognitifs pertinents à l'apprentissage visé. L'analyse des recherches démontre que la construction des CC faite dans la perspective « constructiviste » de l'apprentissage, telle que proposée par Novak, se traduit souvent par une conception selon laquelle les étudiants doivent réaliser leurs cartes de façon « libre » et «non guidée ». L'approche constructiviste s'appuie aussi sur l'idée selon laquelle il n'existe pas de structure « objective » et « prédéterminée » des connaissances qui puisse ou doive être représentée dans la CC. Elle se concrétise, de façon plus ou moins explicite, dans les préférences pour une construction idiosyncrasique des CC exprimant l'organisation des connaissances propre à chaque apprenant, basé sur l'apprentissage par découverte ou " minimalement guidé » ${ }^{[2]}$.

Cependant, les théories pédagogiques d'inspiration socioconstructiviste, tout comme la théorie de la charge cognitive, représentative de la perspective cognitiviste contemporaine, avancent divers arguments en faveur d'un meilleur soutien pédagogique dans les domaines complexes ou pour répondre aux besoins des étudiants possédant peu de connaissances préalables ${ }^{[13]}$. Dans cette perspective, certains chercheurs proposent de mieux soutenir la construction des CC. Parmi les formes du soutien proposées figurent la rétroaction verbale de la part des enseignants durant l'activité de la construction des CC, l'offre des « cartes à trous » et des cartes « squelettes » à compléter par les étudiants. L'enseignant peut aussi prédéterminer les concepts-clés et le type de liens ou adapter la structuration hiérarchique des connaissances dans la CC aux caractéristiques des connaissances d'un domaine, en privilégiant par exemple la structure causale en physiologie ${ }^{[4]}$.

L'importance d'un soutien adéquat des étudiants a également été relevée dans l'APP ${ }^{[8]}$. Les études sur la construction des CC dans le cadre de l'APP indiquent qu'elle est utilisée pour améliorer la génération des hypothèses explicatives, développer la pensée critique, favoriser l'organisation des connaissances et l'apprentissage autodirigé. On constate ainsi que la $\mathrm{CC}$ peut viser des objectifs pédagogiques divers en APP et que les études existantes ne permettent pas de formuler des recommandations sur la façon d'aligner l'activité et les objectifs spécifiques visés.

\section{Objectifs}

Notre recherche s'appuie sur le postulat selon lequel l'alignement pédagogique favorisant la cohérence entre les activités d'apprentissage (ici la construction de la CC), les objectifs visés et les stratégies d'évaluation, améliore l'apprentissage dans une situation donnée.

Dans ce projet, nous avons choisi de nous intéresser à la question de l'organisation des connaissances souhaitée par les responsables des cours d'APP du niveau pré-clinique dans la formation médicale du premier cycle par rapport aux objectifs pédagogiques de leurs cours. Existe-t-il, du point de vue des enseignants, une $\mathrm{CC}$ «idéale » pour l'apprentissage, et si oui, en quoi consiste-t-elle ? Soulignons que cette CC « idéale » n'est pas une carte réalisée pour représenter la vision d'un « expert » du domaine, mais bien une représentation qui devrait «idéalement» être élaborée par les apprenants de façon à favoriser leur apprentissage.

Plus précisément, le but de ce projet est de déterminer s'il existe une conception commune partagée par les enseignants responsables de cours d'APP : 1) des objectifs pédagogiques de l'activité de la construction de la CC et 2) de la nature et de l'organisation des connaissances qui devraient être représentées dans une carte conceptuelle pour un 
cas-problème donné, étant donné les objectifs pédagogiques visés dans le cours.

\section{Méthodes}

Notre méthode consiste en une étude de cas interprétative, visant à décrire et à interpréter théoriquement le phénomène étudié. Il s'agit d'une étude de cas exploratoire, puisque notre objet d'étude présente de nombreuses variables en jeu qui ne sont pas prises en considération à ce stade de la recherche ${ }^{[14]}$.

Une étude pilote a été réalisée afin de préciser la question de recherche. Cette étude a été menée avec deux enseignants cliniciens (dont la chercheure principale) remplissant les critères de sélection des participants de la recherche (décrits ci-dessous). Elle a consisté en une discussion et une analyse des CC des enseignants et des étudiants. Cette étude pilote nous a permis ensuite de mettre au point : a) les instruments de recueil et d'analyse des données, à savoir la conception et le test du déroulement d'une séance de construction de $\mathrm{CC}$ et de son enregistrement en vidéo ; b) un guide d'entretien ; c) une méthode de constitution des protocoles des enregistrements vidéo et d'analyse des données verbales.

\section{Participants}

Nous avons choisi d' analyser les conceptions des enseignants responsables de cours d'APP car d'une part, ceux-ci sont des experts du contenu d'enseignement et car d'autre part, ils jouent (en collaboration avec les tuteurs impliqués dans leur cours) un rôle déterminant dans la conception et la supervision des activités et des objectifs d'apprentissage dans la formation médicale du premier cycle.

Huit enseignants volontaires ont participé à l'étude. Ils ont été choisis parmi les responsables de cours obligatoires du niveau préclinique utilisant l'APP selon les critères suivants : avoir expérimenté l'utilisation des CC dans leur cours et posséder une formation de base en pédagogie. La représentation des différents points de vue a été assurée par la sélection d'un nombre équivalent d'enseignants impliqués en première et en deuxième année du cours de médecine, parmi lesquels trois étaient des chercheurs des sciences fondamentales et cinq des cliniciens. L'attitude des enseignants envers les CC n'a pas fait l'objet d'une évaluation préalable à l'étude et elle n'a pas constitué un critère de sélection des participants.

\section{Recueil de données}

Nous avons utilisé quatre méthodes de recueil de données : 1) la verbalisation concomitante réalisée par chaque participant durant la séance de construction de la CC ; 2) l'entretien semi-dirigé de groupe, à la fin de la séance ; 3) l'observation directe de la séance et 4) le recueil des cartes ou autres traces produites durant la séance.

La CC portait sur un cas-problème choisi par chaque participant parmi les problèmes de son cours.

La verbalisation concomitante constitue un moyen d'accès aux connaissances complexes mobilisées par les sujets dans l'activité et qui peuvent être formulées verbalement ${ }^{[15]}$. Nous l'avons situé dans le cadre d'une séance à laquelle ont participé une dyade de participants, une chercheure experte des représentations graphiques et une clinicienne-chercheure. En tout, quatre séances de verbalisation concomitante ont été réalisées, soit une par dyade de participants.

Chaque séance avait pour objectif le partage des conceptions personnelles sur la carte conceptuelle attendue des étudiants à l'étape du « retour ». Chaque participant devait construire la carte de son cas et décrire à haute voix ce qu'il était en train de faire, en s'adressant à son collègue. Celui-ci avait pour consigne de l'écouter et de poser, lorsqu'il en éprouvait le besoin, des questions sur l'activité de CC en cours. Pour éviter que les discussions durant les séances ne se centrent trop sur des besoins de clarification des contenus scientifiques des cas-problèmes et empêchent ainsi les discussions sur la CC, les dyades étaient composées de participants aux expertises médicales ou disciplinaires connexes.

Une période d'environ 40 minutes était allouée à la construction de la CC par chaque participant, au moyen de l'outil CMap Tools ou d'un tableau blanc et de crayons de couleur. Durant l'activité d'élaboration de la $\mathrm{CC}$, deux chercheures (NCF, BP) ont 
observé l'activité et les échanges en prenant des notes. Après la réalisation de la $\mathrm{CC}$, les deux participants étaient invités à prendre part à un entretien semidirigé de groupe. Cet entretien, conduit par l'une des chercheures, à l'aide du guide d'entretien et des notes d'observation, visait à recueillir les opinions des participants sur leurs conceptions de la $\mathrm{CC}$, la CC réalisée et les objectifs pédagogiques visés dans le cadre de l'APP.

Les quatre séances, d'une durée totale de deux heures, ont eu lieu dans un laboratoire équipé pour réaliser un enregistrement vidéo.

\section{Analyse des données}

Les verbalisations, les échanges réalisés pendant les séances et les entretiens de groupe ont été intégralement retranscrits. L'analyse de ces données a consisté en une analyse thématique à l' aide du logiciel d'analyse qualitative NVivo. Un enregistrement de la verbalisation concomitante a été exclu de l'analyse en raison d'un problème technique.

L'analyse a consisté à élaborer un schème de codage initial, puis à l'enrichir, de façon inductive, sur les verbatims suivants. Elle a permis de dégager trois «niveaux » de la verbalisation concomitante :

- la description de l'activité de la construction de CC (ex. «Je mets les indices dans les bulles»; «Ça, c'est un mot de liaison ») ;

- la description et l'explication du contenu de la CC (ex. "C'est l'interaction entre le surpoids et ces facteurs génétiques, qui vont effectivement expliquer pourquoi cette jeune fille...»; «Donc, on va prendre la sténose, et on va la mettre avant l'AVC»;

- la prescription des caractéristiques de la CC du point de vue de l'enseignant-participant (ex. «Toutes ces étapes doivent faire partie de la carte conceptuelle »; "Il faut vraiment que ça montre ce qui a causé les lésions, jusqu'aux lésions finales, c'est ça qu'on veut »).

Les exemples rapportés ci-haut illustrent l'interaction étroite entre le « dire » et le « faire » dans la verbalisation concomitante à l' activité de la construction de la CC, se traduisant notamment par l'usage des déictiques ( « ici », « ce lien-là »). Pour coder adéquatement les verbatims, les chercheures ont visionné simultanément les enregistrements vidéo de l'activité de la CC. Au besoin, elles ont aussi eu recours aux cartes produites par les participants.

L'analyse des verbatims des entretiens a permis d'enrichir les schèmes de codage en ce qui concerne le contexte de l'APP, les perceptions de l'activité de la CC réalisée par les étudiants et par les tuteurs, et les objectifs pédagogiques visés dans le cours.

Les codes récurrents ont été agrégés en thèmes. La segmentation et la thématisation ont été réalisées de façon récursive sur l'ensemble des verbatims de façon conjointe et collaborative par les deux chercheures qui ont réalisé le recueil des données. Ces deux chercheures ont mobilisé dans l'analyse leurs perspectives et expertises complémentaires : celle de clinicienne, enseignante et responsable des cours de l'APP en médecine et celle de chercheure-enseignante en psychologie de l'éducation, spécialiste du domaine de l'apprentissage avec les CC. Le point de saturation des données a été constaté lors de l'analyse des données de la troisième séance, provenant du cinquième et du sixième participant.

Les résultats de l'analyse thématique ont été discutés avec la troisième chercheure, également enseignante et possédant une expertise sur les processus cognitifs du développement de l'expertise et du raisonnement clinique en médecine (et troisième auteure de cet article), jusqu'à l'obtention du consensus.

\section{Résultats}

La majorité des participants considèrent que la construction des CC peut aider les étudiants à apprendre de façon active mais considèrent que pour que ces bénéfices soient réels, elle doit être «bien utilisée ». Cependant, les participants rencontrent des difficultés à expliquer en quoi consiste cette «bonne utilisation » et ils reconnaissent que les consignes de la méthode de Novak ne les aident pas à le préciser. Lors des séances, les participants ont pu expliciter leurs conceptions en se mettant spontanément «à la place des étudiants ». Ainsi, ils ont constaté que 
«faire la carte est un exercice difficile » et que construire une $\mathrm{CC}$ « complète » ne semble pas constituer un objectif pédagogique approprié à l'étape de « l'aller » car, à cette étape-là, les étudiants n'ont pas les connaissances requises pour relier dans une seule carte « une grande quantité des phénomènes nouveaux », et cela, même de façon «naïve ». En même temps, ils reconnaissent qu'au fur et à mesure de leur avancement dans le programme, les étudiants sont mieux outillés en termes de connaissances et qu'ils arrivent à mieux représenter l'ensemble de phénomènes et leurs relations dans une seule carte. Cependant comme la complexité des cas augmente également avec la progression dans le programme, la construction des CC semble conserver un degré de difficulté considérable, même pour les étudiants les plus avancés dans le programme. Tous les participants se sont dits préoccupés par le manque de temps pour réaliser la CC en groupe, notamment à « l'aller». Ils considèrent aussi que l'utilisation du CMap Tools restreint la possibilité «d'aller au tableau comme dans le bon vieux temps » pour dessiner, faire les diagrammes, représenter des courbes, etc. Les avantages mentionnés de l'outil CMap sont la facilité de modification et la présentation visuelle attrayante.

\section{Objectifs pédagogiques visés par la construction} de la carte conceptuelle

Les participants s'accordent sur le fait qu'une «bonne carte » est celle qui explique le « pourquoi » de la survenue du problème de santé principal et, éventuellement, des problèmes secondaires. L'explication attendue doit être fondée sur les connaissances biomédicales décrivant les «mécanismes d'action » sous-jacents aux phénomènes qui ont conduit au problème de santé principal et à ses manifestations.

Ils trouvent que la CC doit être «contextualisée », c'est-à-dire « centrée sur le patient », sans pour autant reproduire ce qui est décrit dans le texte du cas. Ainsi, une CC qui décrit principalement ce qui est arrivé au patient (son « histoire »), appelée « chronologique » ou « linéaire », ne répond pas aux objectifs pédagogiques. Pour eux, le «contexte» de la carte correspond plutôt au domaine des connaissances biomédicales pertinent par rapport au «cas ». Lors des séances, les participants se sont questionnés sur la quantité des connaissances qui devraient être représentées. Ils s'entendent pour dire qu'une CC trop détaillée indique que l'étudiant n'est pas parvenu à synthétiser ce qui est pertinent. Par exemple, il n'est pas approprié de lister dans la CC toutes les bactéries qui peuvent causer une infection donnée. Plusieurs participants ont proposé de délimiter la «profondeur » des CC à l'aide de « la base de connaissances commune », soit dans une perspective disciplinaire (par ex. les concepts fondamentaux en neurologie), soit dans une perspective «professionnelle » (ce qu'un "médecin généraliste doit savoir » pour être compétent dans sa pratique médicale).

\section{L'organisation attendue des connaissances dans la CC.}

Tous les enseignants ont abordé la construction de la CC comme une tâche d'explication de la structure causale reliant la pathologie aux données cliniques du cas. Les traits communs des $\mathrm{CC} \mathrm{du}$ « retour » sont les suivants :

- Le concept principal est placé au centre de la carte dans sa partie supérieure. Ce concept représente la pathologie principale présentée dans le cas-problème.

- Les concepts cliniques repérés dans le texte sont représentés en bas ou en périphérie de la carte.

- Les concepts cliniques sont organisés en fonction de leur appartenance aux «catégories cliniques », soit les facteurs de risque, les signes, les symptômes, les investigations et les traitements, leurs effets bénéfiques, leurs effets indésirables et les diagnostics secondaires.

- Les éléments cliniques intégrés à la carte ont été transformés sémantiquement (ex. «ménorragies » au lieu de « règles abondantes »).

- Lorsque les éléments cliniques peuvent être expliqués par un même mécanisme, les participants les ont regroupés dans un seul « nœud ».

- La signification verbalisée de la majorité des liens est celle de la causalité (A cause B) et elle peut être exprimée par des mots différents (ex.: A cause, entraîne, provoque B). L'utilisation des verbes plus spécifiques que «cause » est souhaitée pour 


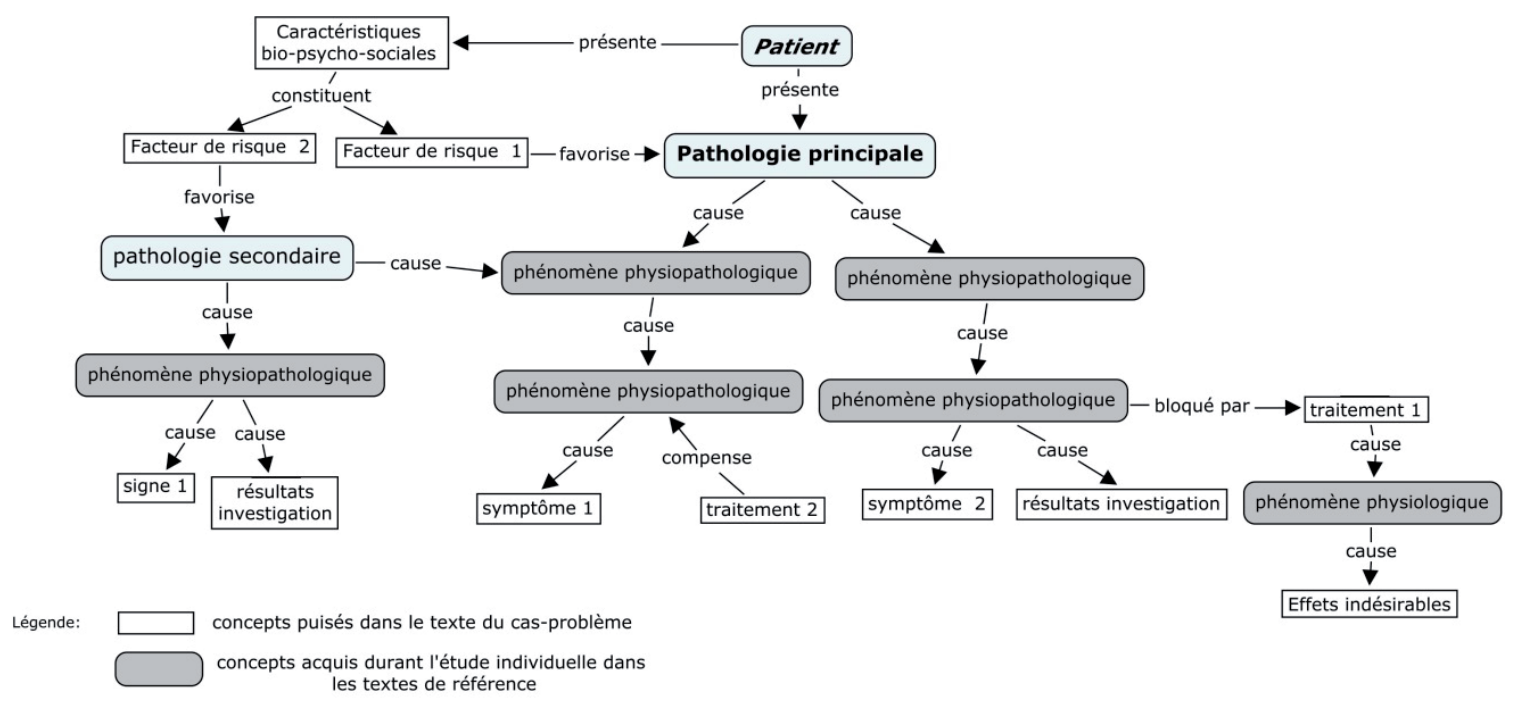

Fig. 2. Le modèle de la carte conceptuelle attendue des étudiants à la phase du « retour » de l'APP.

exprimer soit la terminologie spécifique à un domaine disciplinaire, (par ex., en endocrinologie on retrouve fréquemment les verbes «inhibe» et «stimule »), soit la terminologie «médicale» («favorise $)$.

- Les liens entre les concepts biomédicaux et les concepts cliniques forment des « cascades physiopathologiques ».

- La relation de causalité est habituellement représentée dans la carte sous la forme d'une relation orientée de la « cause » vers «l'effet». Parfois, les participants ont choisi de l'exprimer en utilisant la forme passive d'un verbe de causation ( $\mathrm{B}$ est causé par $\mathrm{A} »)$, représentée aussi par l'orientation du lien « du bas vers le haut»).

- Certains liens expriment l'idée d'une temporalité propre au domaine au moyen de verbes tels que « $s e$ complique », «évolue », «s'améliore ». Des mots exprimant une temporalité plus générale tels que «après » ou « ensuite », sont jugés inadéquats.

Soulignons que les enseignants se sont efforcés de respecter les contraintes de l'organisation hiérarchique des connaissances, telle que proposées par la méthode de Novak, en réorganisant progressivement la carte de façon à placer la "pathologie » qui est le concept le plus général en haut et les concepts les plus spécifiques (les éléments cliniques observés) en bas de la carte (voir figure 2).

\section{Discussion}

Cette étude a permis à un groupe d'enseignants responsables des cours du niveau préclinique d'expliciter leurs représentations des objectifs visés par les $\mathrm{CC}$ et leurs attentes quant à l'organisation des connaissances dans les CC construites par les étudiants analysant un cas-problème au cours de l'APP. Elle leur a aussi permis d'exprimer diverses préoccupations d'ordre pédagogique quant à la façon d'utiliser la CC. Nos résultats indiquent que ces enseignants partagent une représentation commune des objectifs pédagogiques de la construction des CC : elle devrait permettre aux étudiants de construire une représentation cohérente des mécanismes causaux à l'œuvre dans la pathologie ou le problème clinique principal du cas. Pour nos participants, une « bonne carte » est par conséquent celle qui représente la « bonne » organisation des connaissances causales, permettant d'expliquer correctement et de façon aussi complète que possible l'ensemble des observations cliniques décrites dans le cas-problème.

Les enseignants qui ont participé au projet accordent une grande importance à la compréhension des mécanismes causaux d'une pathologie particulière mais aussi des principes généraux structurant un domaine (ex. voies neurologiques, boucle de rétroaction en endocrinologie). Ils ne souhaitent pas que les 
étudiants relient les données cliniques au moyen d'inférences de type clinique puisque cela ne leur permet pas de s'assurer que les étudiants aient suffisamment développé leurs connaissances biomédicales en lien avec le cas-problème. Par exemple, pour un casproblème dont l'objectif pédagogique serait « expliquer les manifestations cliniques des pneumonies », il ne serait pas adéquat qu'un étudiant relie pneumonie et toux par un lien de type « se manifeste » sans le développer davantage. L'organisation hiérarchique causale des connaissances dans la CC est cohérente avec l'objectif pédagogique des deux premières années du préclinique, qui est celui d'aider les étudiants à développer une base de connaissances théoriques utiles pour la résolution de problèmes cliniques.

Nos résultats vont dans le sens des recherches qui soulignent l'importance de l'organisation causale des connaissances biomédicales et leur « pouvoir intégrateur » dans l'apprentissage des étudiants du niveau préclinique $^{[16]}$. Woods et ses collègues ${ }^{[17]}$ proposent que ce soit précisément la structure causale des connaissances biomédicales en lien avec une pathologie donnée qui assure la cohérence et la stabilité du cadre explicatif de phénomènes cliniques perçus. La structure causale permet d'organiser de façon cohérente les connaissances cliniques, de les retenir et d'amorcer la création de scripts cliniques. Ces idées sont appuyées par l'ensemble des travaux sur le développement de l'expertise médicale qui démontrent que les connaissances biomédicales « encapsulées » jouent un rôle essentiel dans la résolution des problèmes cliniques complexes ou rares ${ }^{[18]}$

Dans le cadre d'une démarche participative de la révision du programme, l'étude a été présentée à l'ensemble des responsables de cours de l'APP (y compris les participants à l'étude) $(n=14)$, aux responsables du programme $(n=5)$ ainsi qu'à une représentante académique des étudiants. L'ensemble des personnes présentes ont accueilli favorablement les résultats en estimant que ceux-ci répondaient en grande partie à leurs interrogations sur la CC en APP. Les responsables de cours ont trouvé que l'étude a permis de clarifier l'objectif de la construction de la $\mathrm{CC}$ en APP, ce qui les aidera à mieux ajuster cette activité aux objectifs d'apprentissage. La représentante des étudiants a exprimé un certain soulagement à l'idée que les étudiants disposeraient dorénavant de consignes plus précises sur la CC. Quant aux responsables de programme, ils ont pu situer l'activité de construction de la CC dans une perspective plus globale d'acquisition de l'expertise et mettre sur pied des formations pédagogiques intégrant les résultats de notre étude et permettant de préciser l'utilisation de la CC en APP au cursus préclinique. Depuis, ces formations ont été offertes à environ 150 tuteurs et 250 étudiants. Elles ont donné l'occasion de confronter nos résultats à la réalité du « terrain », et de constater que notre étude a permis d'expliciter et de clarifier les opinions et les pratiques implicitement partagées, tant par des étudiants que par des enseignant, quant à la structure et le contenu souhaitables de la CC. Par contre, ces formations ont aussi mis en évidence qu'il demeure encore des questions sur l'apport de la CC comme outil de collaboration et d'animation dans une séance d'APP.

L'ensemble de ces résultats nous permettent donc de formuler plusieurs recommandations pour favoriser l'alignement de l'activité de la CC et des objectifs pédagogiques dans le cadre de l'APP tel qu'il est développé dans le cursus préclinique.

Tout d'abord, il est souhaitable de mieux guider les étudiants en clarifiant les consignes de la construction des CC quant aux objectifs pédagogiques, l'organisation attendue des connaissances dans la $\mathrm{CC}$, la nature des connaissances cliniques (symptômes, signes, traitements, facteurs de risque, etc.) et biomédicales (causes) représentées. Une meilleure représentation de la tâche à réaliser peut contribuer à diminuer la charge cognitive « inutile » ${ }^{[19]}$, résultant des questionnements sur la façon de réaliser la tâche (comment je m'y prends, qu'est-ce qui est attendu de moi ?) et à diriger les ressources cognitives des étudiants vers les activités de compréhension du domaine à l'étude, notamment à la phase de l'étude individuelle.

Ce cadre commun de compréhension des objectifs de la représentation des connaissances dans une CC peut aussi favoriser la rétroaction des tuteurs. Mieux outillés, les tuteurs peuvent plus facilement s'assurer d'une compréhension commune du casproblème, détecter les erreurs ou les omissions 
importantes et évaluer les cartes produites individuellement.

Nos résultats peuvent aussi être utilisés pour aider les enseignants responsables des cours de l'APP à mieux situer l'utilisation de la $\mathrm{CC}$ en fonction des objectifs d'apprentissage du cas-problème, en distinguant ceux qui gagneraient à faire l'objet de l'activité de la construction de la $\mathrm{CC}$, ce qui favoriserait les ajustements quant à l'ampleur et la complexité de la $\mathrm{CC}$ en fonction du niveau des connaissances des étudiants. On pourrait par exemple proposer, en utilisant la taxonomie de Bloom ${ }^{[20]}$, que seuls les contenus en lien avec les objectifs de type « expliquer » ou « comprendre » devraient faire l'objet de la CC.

Les discussions menées dans le cadre des formations pédagogiques sur les $\mathrm{CC}$ ont permis de confirmer nos résultats et de documenter que l'activité consistant à relier l'ensemble de phénomènes d'un cas-problème dans la carte, sans avoir bâti un bagage de connaissances suffisant sur la pathologie exemplifiée, constitue un exercice complexe et difficile à réaliser dans le cadre de la durée habituelle d'une séance d'APP à la phase « aller ». Il semble que les processus de production d'une représentation externe « complète », répondant aux critères de forme d'une $\mathrm{CC}$ réalisée avec le logiciel CMap Tools, monopolisent le travail cognitif des étudiants au détriment des processus d'activation des connaissances antérieures, de leur partage et de génération des hypothèses, parfois erronées mais riches en termes d'apprentissage.

En d'autres mots, la tâche de «faire la carte » devient l'objectif principal des étudiants, diminuant le temps et l'attention consacrés à la discussion de leurs connaissances et à la génération des hypothèses explicatives, activités qui constituent pourtant le but premier de cette phase de l'APP. Certaines observations réalisées par les enseignants permettent même d'avancer que la représentation des hypothèses sur les relations entre les phénomènes sous la forme de liens dans la CC conduit à figer la représentation du problème sous une forme unique et freine la discussion en obligeant le groupe à trouver un consensus, alors que ses membres n'y sont pas encore prêts.

C'est pourquoi nous proposons de réserver la construction d'une CC « complète » aux phases de l'étude individuelle et du « retour » de l'APP. Pour améliorer l'atteinte des objectifs de l'APP à la phase « aller », nous pensons qu'il est préférable que les étudiants puissent générer des hypothèses partielles concernant les causes de certains éléments cliniques ou parfois regrouper certains éléments, sans pour autant les relier sous la forme d'une CC unique. De plus, considérant nos résultats à la lumière de l'ensemble des recherches sur l'apprentissage soutenu par les représentations externes des connaissances $^{[1]}$, nous déconseillons l'imposition de la CC à titre d'une représentation externe unique à cette étape de l'APP. Au contraire, les étudiants et les tuteurs devraient avoir le choix des représentations graphiques congruentes avec le domaine à l'étude ; par exemple, ils devraient pouvoir utiliser un dessin de circuits neuronaux pour expliquer les signes cliniques d'un patient ayant eu un accident vasculaire cérébral.

Pour assurer la fiabilité et la crédibilité de notre étude de cas, nous avons apporté une attention particulière à la représentativité des participants, dont le nombre et certaines caractéristiques ont été prédéterminés afin d'assurer la diversité des points de vue. Cette démarche s'est avérée satisfaisante étant donné la saturation des données obtenue à l' analyse des protocoles. Nous avons par ailleurs triangulé nos sources de données, nos méthodes de recueil et les perspectives des chercheures lors de l'analyse et de l'interprétation des résultats. La crédibilité de notre étude a également été augmentée par la validation des résultats par d'autres enseignants impliqués dans les cours APP en formation préclinique.

La transférabilité de nos résultats pourrait être améliorée au moyen d'une démarche de généralisation analytique ${ }^{[21]}$ visant à les comparer aux résultats d'autres recherches. Ainsi, il serait souhaitable d'étudier l'influence de diverses variables relatives à l'APP, par exemple, les objectifs visés par les différentes composantes de l'APP, la structuration des casproblèmes ou le rôle des tuteurs. Dans notre contexte d'utilisation de l'APP, la CC est perçue d'abord comme un « outil cognitif » qui devrait favoriser l'organisation causale des connaissances biomédicales dans le domaine visé par le cas-problème. Son potentiel en tant qu' " outil métacognitif », visant à soutenir l'autorégulation des étudiants, ne s'est pas reflété dans nos résultats et devrait, par conséquent, 
faire l'objet d'autres études. Nos résultats devraient également être comparés à ceux issus des recherches considérant le point de vue des étudiants et leur activité de la construction de la CC.

Une limite de notre recherche est qu'elle se concentre sur le produit de la construction de la CC, puisque, malgré la prise en compte des actions de construction de la CC, notre analyse n'a pas visé à déterminer les processus de raisonnement impliqués. Ainsi, elle laisse ouverte la question de la directionnalité du raisonnement lors de la construction de la CC (du diagnostic vers les données cliniques ou inversement). Or, cette question est particulièrement importante en ce qui concerne la préparation des étudiants à l'apprentissage du raisonnement clinique. Pour mieux comprendre les apports potentiels du soutien offert par des représentations externes à l'organisation des connaissances biomédicales dans le cadre de l'APP et leur rôle dans l' apprentissage du raisonnement clinique, une perspective intéressante serait de comparer les processus de construction des $\mathrm{CC}$ par les experts (les enseignants) et par les novices (étudiants), à différentes étapes de leur formation.

\section{Contributions}

Natalie Caire Fon et Béatrice Pudelko ont participé à la conception du protocole de recherche, au recueil et à l'analyse des données, à l'interprétation des résultats et à l'écriture du manuscrit ; MarieClaude Audétat a participé à l'analyse des données, à l'interprétation des résultats et à l'écriture du manuscrit.

\section{Approbation éthique}

Cette étude a obtenu le certificat d'éthique de l’Université de Montréal.

\section{Déclaration d'intérêts}

Aucun auteur ne déclare de conflit d'intérêt en lien avec le contenu de cet article.

\section{Subvention}

Cette étude a bénéficié d'une subvention de recherche accordée par le Fonds institutionnel de recherche de la Télé-université et de l'aide logistique du Centre de recherche LICEF.

\section{Remerciements}

Nous remercions le docteur Serge Dubé pour sa participation à l'étude pilote, les professeurs de la Faculté de médecine de l'Université de Montréal pour leur contribution volontaire à cette étude et le personnel technique du Centre de recherche LICEF pour son aide à la réalisation du recueil de données.

\section{Références}

1. Jonassen DH, Marra RM. Concept mapping and other formalisms as mindtools for representing knowledge. Association for Learning Technology Journal 1994;2:50-6.

2. Novak JD. Concept mapping: a useful tool for science education. J Res Sci Teach 1990;27:937-49.

3. Chang Y-H, Chang C-Y, Tseng Y-H. Trends of science education research: An automatic content analysis. J Sci Educ Technol 2010;19:315-31.

4. Pudelko B, Young M, Vincent Lamarre P, Charlin B. Mapping as a learning strategy in health professions education: a critical analysis. Med Educ 2012;46: 1215-25.

5. Demeester A, Vanpee D, Marchand C, Eymard C. Formation au raisonnement clinique: perspectives d'utilisation des cartes conceptuelles. Pédagogie Médicale 2010;11:81-95.

6. Daley BJ, Torre DM. Concept maps in medical education: an analytical literature review. Med Educ 2010;44:440-8.

7. Biggs J. Enhancing teaching through constructive alignment. High Educ 1996;32:347-64.

8. Schmidt HG, Rotgans JI, Yew EH. The process of problem based learning: what works and why. Med Educ 2011;45:792-806. 
9. Zwaal W, Otting H. The impact of concept mapping on the process of problem-based learning. Interdisciplinary Journal of Problem-based Learning 2012;6:7.

10. Gijselaers W. Perspectives on problem-based learning. New York (NY) : Springer, 1995.

11. Institute of Human and Machine Cognition (IHMC). Pensacola (FL). CMap Tools software and research. 2016 [On-line] Disponible sur : http://cmap.ihmc.us/

12. Rochette A, Bélisle M, Laflamme A, Doucet M, Chaput M, Fillion B. Étude descriptive de l'utilisation des cartes conceptuelles comme stratégie pédagogique en sciences de la santé. Pédagogie Médicale 2010;11:97-109.

13. Tobias S, Duffy TM. Constructivist instruction: Success or failure? New York (NY): Routledge, 2009.

14. Merriam SB. Case study research in education: A qualitative approach. San Francisco (CA): JosseyBass; 1988.

15. Klein G, Hoffman RR. Macrocognition, mental models, and cognitive task analysis methodology. In: Schraagen JM, Militello LG, Ormerod TC, Lipshitz $\mathrm{R}$, editors. Naturalistic decision making and macrocognition. Hampshire (UK): Ashgate Publishing; 2008, 57-80.
16. Norman G. Teaching basic science to optimize transfer. Med Teach 2009;31:807-11.

17. Woods NN, Brooks LR, Norman GR. The value of basic science in clinical diagnosis: creating coherence among signs and symptoms. Med Educ 2005; 39:107-12.

18. Charlin B, Boshuizen H, Custers EJ, Feltovich PJ. Scripts and clinical reasoning. Med Educ 2007;41: 1178-84.

19. Young JQ, Van Merrienboer J, Durning S, Ten Cate O. Cognitive Load Theory: Implications for medical education: AMEE Guide No. 86. Med Teach 2014; 36:371-84.

20. Anderson LW, Krathwohl DR, Bloom BS. A taxonomy for learning, teaching, and assessing: A revision of Bloom's taxonomy of educational objectives: New York (NY): Adison Wesley Longman, 2001.

21. Yin R. Case study research: Design and methods. Beverly Hills (CA): Sage Publishing, 1994.

Correspondance et offprints: Nathalie Caire Fon, Faculté de médecine, C.P. 6128, succursale Centre-ville.

Montréal QC H3C 3J7, 5143402800 (ext 3514).

Mailto : nathalie.cairefon@umontreal.ca 